\title{
The Role of Microstructure in Luminescent Properties of Er-doped Nanocrystalline Si Thin Films*
}

\author{
M. V.Stepikhova ${ }^{1}$, M. F. Cerqueira ${ }^{2}$, M. Losurdo ${ }^{3}$, M. M. Giangregorio ${ }^{3}$, E. Alves ${ }^{4}$, T. Monteiro ${ }^{5}$ \\ and M. J. Soares ${ }^{5}$ \\ ${ }^{1}$ Institute for Physics of Microstructures, Russian Academy of Sciences, Nizhni Novgorod, 603950 Russia \\ ${ }^{2}$ Departamento de Física, Universidade do Minho, Campus de Gualtar, Braga, 4710-057 Portugal \\ ${ }^{3}$ Institute of Inorganic Methodologies and Plasmas, IMIP-CNR, via Orabona, Bari, 4-70126 Italy \\ ${ }^{4}$ Instituto Técnico Nuclear ITN, EN 10, Sacavém, 2686-953 Portugal \\ ${ }^{5}$ Departamento de Física, Universidade de Aveiro, Campus de Santiago, Aveiro, 3700 Portugal
}

\begin{abstract}
In this contribution, we present a structural and photoluminescence (PL) analysis of Er-doped nanocrystalline silicon thin films produced by rf magnetron sputtering method. We show the strong influence of the presence of nanocrystalline fraction in films on their luminescence efficiency at 1.54 $\mu \mathrm{m}$ studied on a series of specially prepared samples with different crystallinity, i.e., percentage and sizes of Si nanocrystals. A strong increase, by about two orders of magnitude, of Er-related PL intensity in these samples with lowering of the Si nanocrystal sizes from 7.9 to about $1.5 \mathrm{~nm}$ is observed. The results are discussed in terms of the sensitization effect of Si nanocrystals on Er ions.
\end{abstract}

Keywords: Photoluminescence, Erbium, nanocrystalline silicon, ellipsometry

\section{Introduction}

Recently, Er-doped Si materials were widely studied in context with the interest in temperature-stable light emitters for optical communication systems. The intra-4f-shell transitions between the two lowest spin orbit levels of $\mathrm{Er}^{3+}$ ions, namely, the transitions ${ }^{4} \mathrm{I}_{13 / 2} \rightarrow{ }^{4} \mathrm{I}_{15 / 2}$, occur at $1.54 \mu \mathrm{m}$, a wavelength close to that with minimum loss in silica-based optical fibers. The realization of electroluminescence devices on the basis of Si:Er has been reported [1-3]. Nevertheless, the main drawback to using a bulk $\mathrm{Si}$ crystal as a host for $\mathrm{Er}^{3+}$ is the strong temperature quenching of $1.54 \mu \mathrm{m}$ luminescence. It appears that this situation may be considerably improved by the incorporation of Er ions in nanocrystalcontaining materials. The idea is based on the band-gap widening of nanometer-size Si, which consequently has to result in reducing the thermal quenching for Er luminescence. Moreover, Si nanocrystals that are well known to emit in the visible range (due to the recombination of confined excitons within the nanostructures) may act as efficient sensitizers for rare earth ions $[4,5]$

In this contribution, we discuss the luminescent properties of Er-doped nanocrystalline silicon thin films (nc-Si:Er) produced by the rf reactive magnetron sputtering method. The advantages of these films, when compared with the intensively studied nanocrystal-containing $\mathrm{SiO}_{2}$ structures and a$\mathrm{Si}: \mathrm{H}, \mathrm{O}, \mathrm{Er}$ films, is their relatively high conductivity, which makes the material attractive for device applications. One can show that the presence of a crystalline fraction in thin films results in an increase in film conductivity of several orders of magnitude [6]. Er-doped nc-Si:H films with well defined 
crystallinity and nanocrystal sizes (varied from 1-3 to $8 \mathrm{~nm}$ ) were deposited and studied in both the near-IR $(1.54 \mu \mathrm{m})$ and visible luminescence ranges. The results are discussed in terms of the role of $\mathrm{Si}$ nanocrystallites in the luminescent properties of films.

\section{Experimental}

Erbium-doped nanocrystalline silicon thin films were grown by rf magnetron sputtering in an $\mathrm{Ar} / \mathrm{H} 2$ atmosphere on ordinary glass substrates. The procedure applied was similar to that used for the preparation of undoped $\mu \mathrm{c}-\mathrm{Si}: \mathrm{H}$ films in [7], only modified by the introduction of small pieces of metallic erbium to the c-Si target for Er doping. The target used was c-Si of high purity (99.99\%). The erbium was placed in a low erosion area on the silicon target in order to keep a moderate rate of Er impurity. The substrate-target distance was fixed at $55 \mathrm{~mm}$.

Samples with different structural parameters, i.e., different crystalline fraction and grain sizes, were obtained by varying the experimental parameters (RF power, temperature, Er content, and gas-mixture composition). In particular, amorphous films (see Er28) were obtained at low hydrogen dilution, i.e., a low RH value (see Table 1). Nanocrystalline samples were grown in a H2-rich atmosphere, where the role of atomic hydrogen is to etch preferentially the amorphous phase and promote the amorphous-tocrystalline transition. The growth conditions of the films are listed in Table 1.

Table 1. Growth conditions for erbium-doped nanocrystalline silicon thin films

\begin{tabular}{c|c|c|c}
\hline Sample & Temperature, ${ }^{\circ} \mathrm{C}$ & RF power, $\mathrm{W}$ & $R_{H}$ \\
\hline Er22 & 400 & 80 & 0.63 \\
Er19 & 200 & 80 & 0.63 \\
Er24 & 50 & 80 & 0.63 \\
Er28 & 200 & 150 & 0.37 \\
Er33 & 25 & 80 & 0.63 \\
\hline
\end{tabular}

Note: $R_{H}=p \mathrm{H}_{2} /\left(p \mathrm{H}_{2}+p \mathrm{Ar}\right)$ is the hydrogen fraction.

The chemical composition of the films was determined using Rutherford backscattering spectroscopy and elastic recoil detection techniques. The structural characterization was performed using standard micro-Raman spectroscopy under excitation with the $514.5 \mathrm{~nm} \mathrm{Ar}+$ laser line and by x-ray diffractometry in the grazing incidence geometry. For more detailed analysis of the microstructure and film "anatomy", high-resolution transmission electron microscopy (HRTEM) and spectroscopic ellipsometry (SE) were applied. SE spectra of the pseudodielectric function, $\langle\varepsilon\rangle=\left\langle\varepsilon_{1}\right\rangle+i\left\langle\varepsilon_{2}\right\rangle$ were acquired in the 1.5- to 5.5-eV energy range by using a phase-modulated spectroscopic ellipsometer (UVISEL-Jobin Yvon) at an incident angle of $70.5^{\circ}$. SE spectra were analyzed in terms of optical models based on the Bruggeman effective medium approximation (for more details, see [8]). The thickness of the films was evaluated from analysis of the interference pattern in transmission spectra making use of the Swanepoel method [9] and from the spectroscopic ellipsometry data. Photoluminescence (PL) measurements in the near-IR range were performed with a Bruker 66V Fourier-transform spectrometer. The signal was detected with a North-Coast EO-817 liquid nitrogencooled germanium detector. The 514.5-nm line of an Ar laser was used for the excitation. PL studies in the visible spectral range were carried out under excitation with a 325-nm line of a $\mathrm{cw} \mathrm{He}-\mathrm{Cd}$ laser 
with a Spex 1704 monochromator and a cooled Hamamatsu R928 photomultiplier in the detection chain.

\section{Results and discussion}

Figure 1 shows the XRD and Raman spectra for the nc-Si:Er samples studied. The broad band related to the silicon amorphous matrix is present in the spectra for all samples. The Er28 sample grown at higher RF power but in an Ar-rich atmosphere does not show any crystalline peak in either the XRD or Raman spectra (the same behavior was also observed for the Er33 sample). In contrast, the (111), (220), and (311) diffraction peaks of c-Si are visible for all other samples. The peaks are well evident for the Er22 sample grown at high $\mathrm{H}_{2}$ dilution and a high temperature of $400^{\circ} \mathrm{C}$, both parameters promoting the amorphous-to-crystalline transition. The diffraction peaks have a lower intensity for the Er19 and Er24 samples, indicating a decrease in the crystallinity and/or the crystallite grain size, which is also confirmed by the intensity ratio of the Stokes peaks at $480 \mathrm{~cm}^{-1}$ (a-Si related) and at $520 \mathrm{~cm}^{-1}$ (transverse optical (TO) mode of c-Si) in the Raman spectra (Fig. 1b). The diffraction peak analysis, by fitting a pseudo-Voigt function to the (111) c-Si diffraction peak [7], gives the average crystal size for the Si nanocrystals presented in Table 2. In the same table, the data of Raman spectroscopy are also presented. To analyze the Raman spectra, the TO replica of amorphous structure has been approximated by a Gaussian profile and the crystalline response analysis was performed on the basis of the strong phonon confinement model [10].
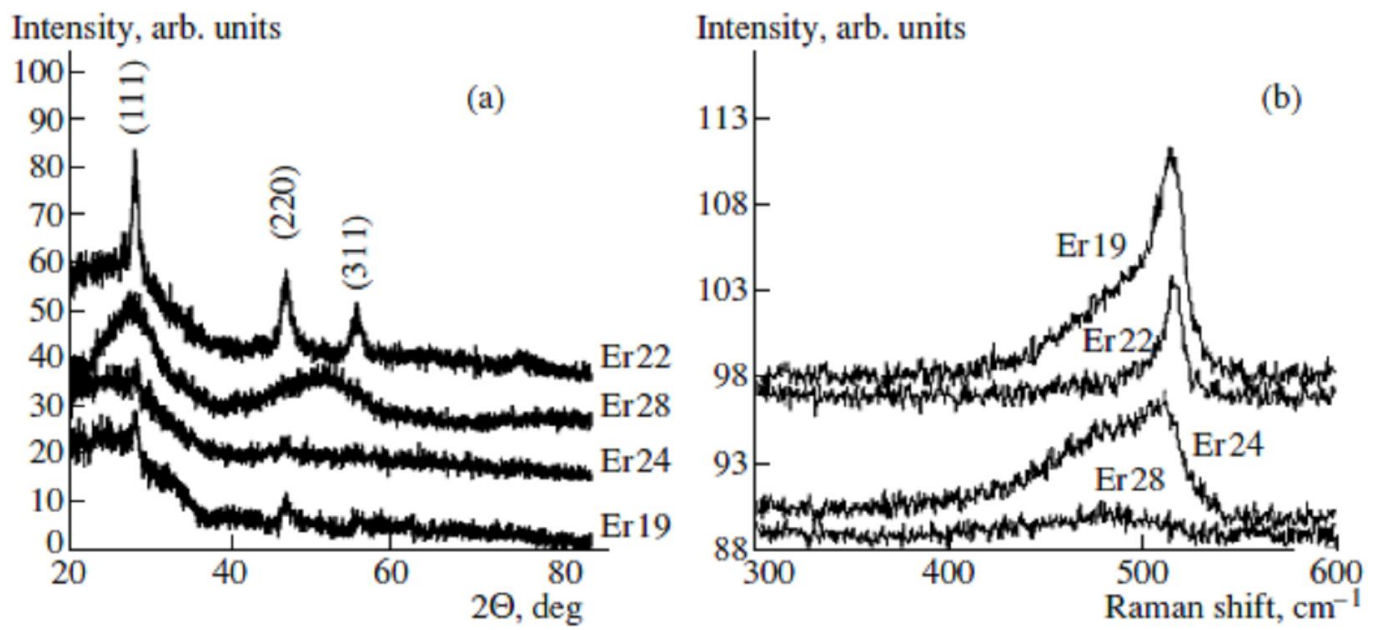

Fig. 1. (a) XRD and (b) Raman spectra of nc-Si : Er thin films.

Table 2. Content of elements, thickness, and structural parameters of nc-Si : Er samples

\begin{tabular}{c|c|c|c|c|c|c|c|c|c}
\hline Sample & Er, $\%$ & $\mathrm{Si}, \%$ & $\mathrm{O}, \%$ & $\mathrm{H}, \%$ & $d, \mathrm{~nm}$ & $D_{X}, \mathrm{~nm}$ & $D_{R}, \mathrm{~nm}$ & $C_{R}, \%$ & $\mathrm{SE}$ data \\
\hline Er22 & 0.10 & 71.7 & 8.8 & 17.6 & 2089 & 7.0 & 7.9 & 65 & $87 \% \mu \mathrm{c}-\mathrm{Si}$ \\
Er19 & 0.12 & 62 & 34 & 23 & 483 & 5.7 & 6.5 & 43 & $31 \% \mu \mathrm{c}-\mathrm{Si}$ \\
Er24 & 0.17 & 56.5 & 17.6 & 25.4 & 538 & 3.9 & 5.5 & 23 & $25 \% \mu \mathrm{c}-\mathrm{Si}$ \\
Er28 & 0.11 & 60.9 & 2.9 & 34.3 & 1295 & - & - & 0 & $10 \% \mathrm{nc}-\mathrm{Si}$ \\
Er33 & 0.02 & 73.4 & $<1$ & 25.8 & 1499 & - & - & 0 & $38 \% \mathrm{nc}-\mathrm{Si}$ \\
\hline
\end{tabular}

Note: $D$ - average crystal size, $R$ - Raman spectroscopy, $X-\mathrm{XRD}$ analysis, $C_{R}$ - crystalline volume fraction determined by Raman spectroscopy, $d$ - film thickness. 
Near-IR photoluminescence spectra measured at $77 \mathrm{~K}$ in nc-Si:Er samples are shown in Fig. 2. Let us consider the highly crystalline samples according to XRD and Raman data (samples Er24, Er19, and Er22 with nanocrystal sizes of 5.5-7.9 $\mathrm{nm}$ and crystallinity $C_{R}=23-65 \%$ ). The spectra of these samples show a luminescence peak at $1.54 \mu \mathrm{m}$ related to the intraatomic $\left({ }^{4} \mathrm{I}_{13 / 2} \rightarrow{ }^{4} \mathrm{I}_{15 / 2}\right)$ transitions of $\mathrm{Er}^{3+}$ ions. Being relatively broad in a low crystalline sample (sample Er24) with a maximum at 6500 $\mathrm{cm}^{-1}$ and a characteristic shoulder at around $6457 \mathrm{~cm}^{-1}$, like for $\mathrm{Er}$ in glasslike and amorphous materials [11], the Er-related spectrum transforms in highly crystalline films (sample Er22) in the spectrum with a fine line structure (see inset A in Fig. 2), giving evidence for the incorporation of Er ions in regular crystalline surroundings. At the same time, in the spectra of highly crystalline samples, the lines at 7500 and $9435 \mathrm{~cm}^{-1}$ appear and increase with the crystallinity and could be assigned following from their energetic position as the defectlike and excitonic transitions in Si crystallites. However, one can see that the increase of crystallinity in these samples results in a strong quenching of Er-related photoluminescence.

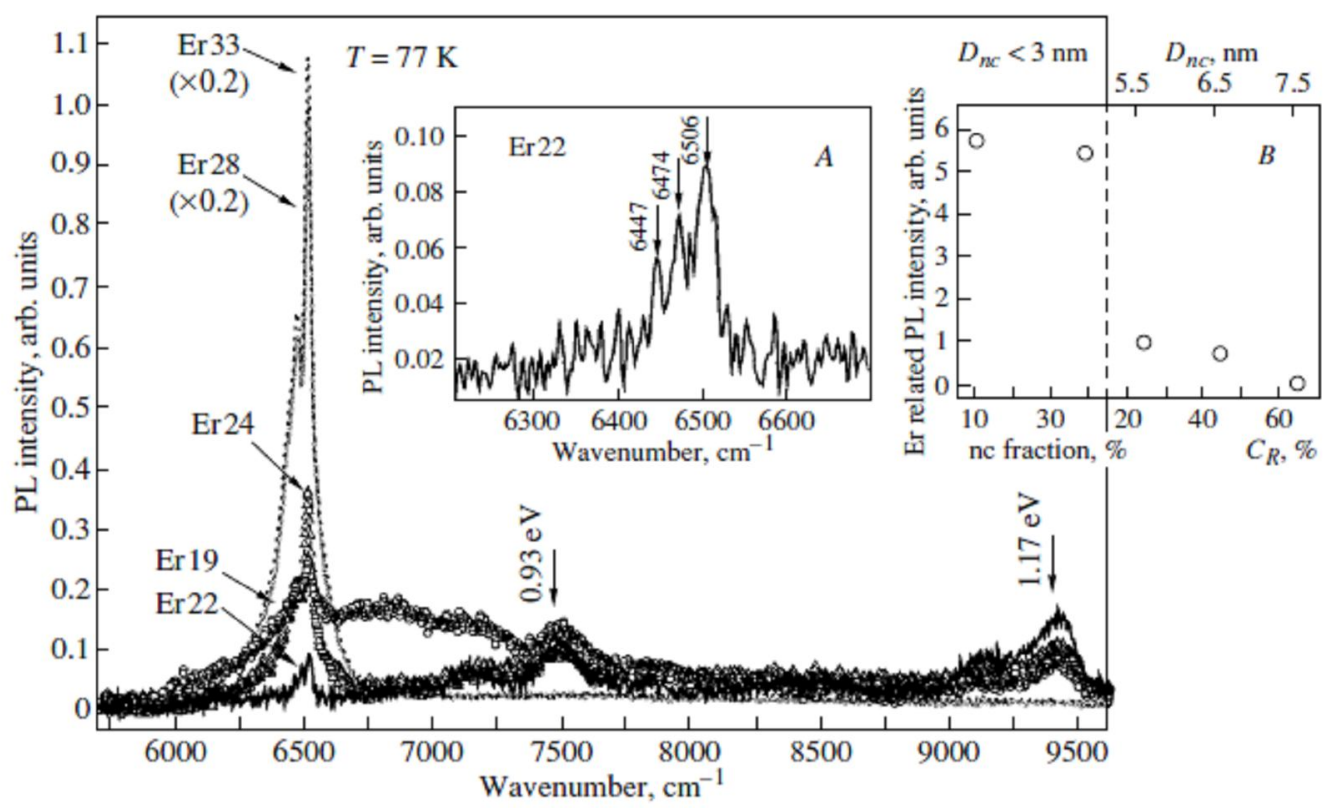

Fig. 2. PL spectra of nc-Si : Er samples with different contents of the crystalline fraction. Insets show $(A)$ the enlarged Er-related region in the PL spectrum for sample $\mathrm{Er} 22$ and $(B)$ the correlation between the PL intensity (Er-related peak) and the presence of a crystalline fraction in nc-Si : Er samples. The right part of inset $B$ shows the situation for highly crystalline samples according to XRD and Raman analysis (the $C_{R}$ and $D_{R}$ values on the bottom and top axes correspond to the Raman data). On the left part of inset $B$, PL intensities for the samples with a nanocrystallite fraction with 1-3 nm crystallite grain sizes are presented, where the values for the nc fraction are taken from SE data. The luminescence intensities at $1.54 \mu \mathrm{m}$ in this inset are normalized to the thickness of the films. 


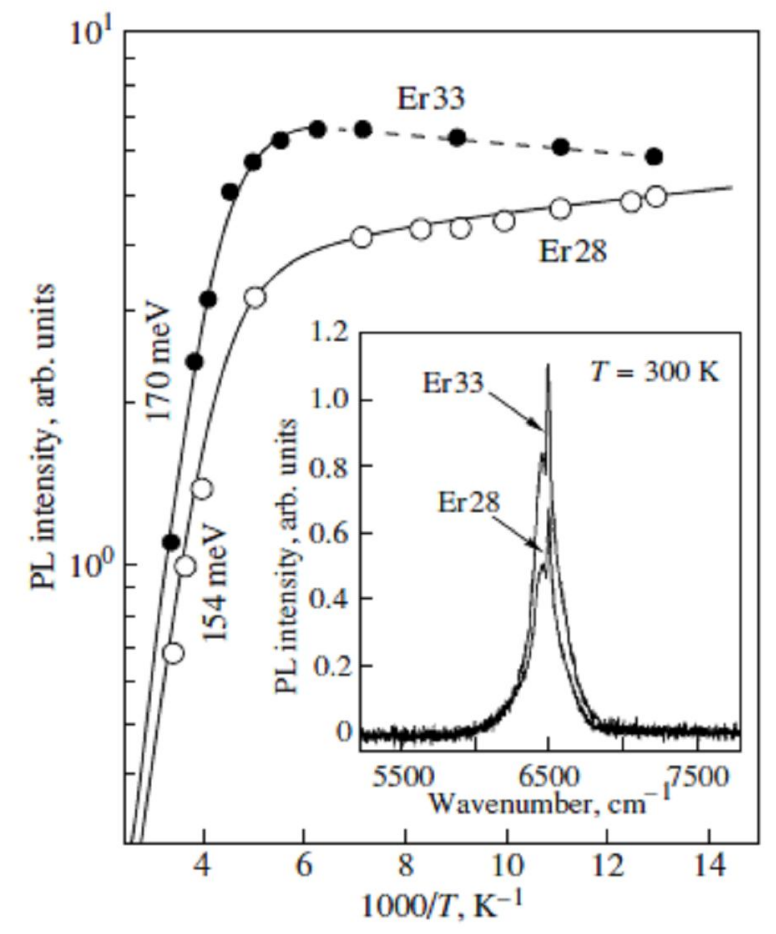

Fig. 3. Temperature dependences of Er-related PL intensity obtained in nc-Si : Er thin films (samples Er28 and $\mathrm{Er} 33$ ).

The solid lines are exponential fits of experimental data with deactivation energies of 170 and $154 \mathrm{meV}$ for samples $\mathrm{Er} 33$ and $\mathrm{Er} 28$, respectively. The inset shows PL spectra of the samples at room temperature.

Though the samples have a similar Er atomic percentage $(0.1-0.17 \%$, as estimated by RBS), the PL intensity in them reduces by more than an order of magnitude with an increase in the crystalline fraction from 23 to $65 \%$ (and crystallite size from 5.5 to $7.9 \mathrm{~nm}$; see inset B in Fig. 2).

The most intense Er-related PL was observed in low crystalline samples determined as "amorphous" according to the Raman and XRD analysis (samples Er28 and Er33 in Table 2). In particular, the PL intensity of the Er33 sample exceeds that for highly crystalline sample Er22 by about two orders of magnitude (see inset to Fig. 2) despite the lower Er content. These samples show strong luminescence at room temperature (Fig. 3). The PL intensity decreases only fivefold when going from 77 to $300 \mathrm{~K}$ with deactivation energies of 154 and $170 \mathrm{meV}$.

It seems that it would be difficult to explain this dramatic increase in luminescent efficiency arising upon the transition from crystalline to amorphous film structure even because of the strong difference in the excitation cross sections for Er ions in these two matrices. It is known that the excitation cross section for the direct excitation of Er ions in amorphous matrices is about five orders of magnitude less than that for Er in crystalline $\mathrm{Si}\left(8 \times 10^{-21}\right.$ and $3 \times 10^{-15} \mathrm{~cm}^{2}$, respectively $\left.[12,13]\right)$. Of course, one cannot exclude the role of nonradiative recombination channels in these composite structures. So, we can image that the enlargement of the crystalline fraction will enhance the nanocrystalline interactions in films and, therefore, the probability of excitons propagating in the crystalline network and recombining nonradiatively. However, in fact, we did not observe any direct evidence of a strong influence of nonradiative processes on the luminescent efficiency of the films. There is no strong correlation 
between the amount of hydrogen in the films and their luminescent properties (as a rule, hydrogen passivates the dangling bonds in amorphous/crystalline tissue, thus intensifying PL).We observed even the opposite, an increase in the film PL intensity after annealing at $500^{\circ} \mathrm{C}$ for $5 \mathrm{~h}$; this procedure depletes the material with hydrogen. Moreover, the presence of oxygen in films, an element known as an "activator" for Er ions, also does not influence noticeably the film luminescence (see Table 2).

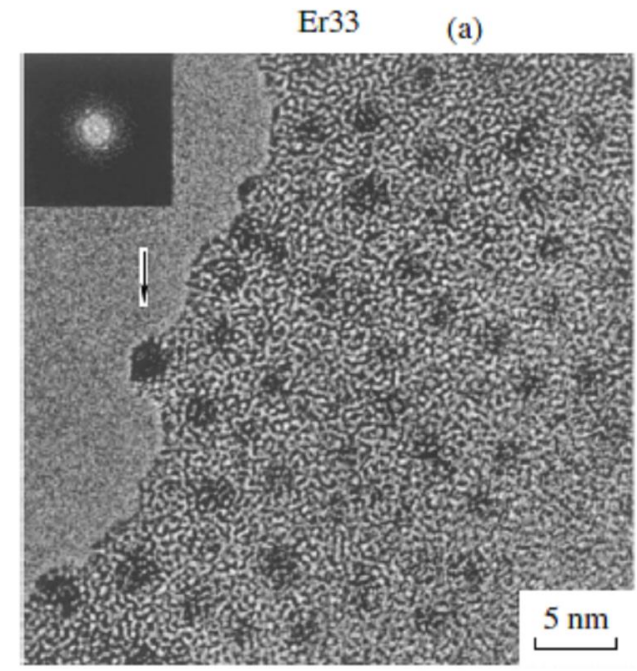

(b)

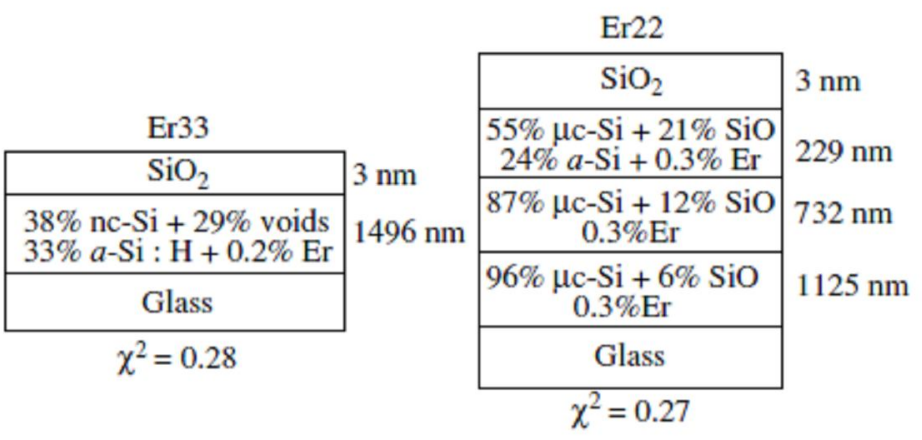

Fig. 4. (a) HRTEM image obtained for sample $\operatorname{Er} 33$ and (b) structural models of samples $\operatorname{Er} 33$ and $\operatorname{Er} 22$ derived from SE analysis.

One can assume that the luminescence of Er ions in "amorphous" samples is activated by the nanocrystals with very small grain sizes $(<3 \mathrm{~nm})$. The first indication of the presence of these nanocrystals in films was obtained in spectroscopic ellipsometry studies. SE data predict the presence of small nanocrystals in samples Er28 and Er33 in an amount of 10 and 38\%, respectively (SE analysis of the nc-Si thin films with small nanocrystal grain sizes were discussed in detail in [14]; see also the models in Fig. 4b). Indeed, this prediction can be confirmed by HRTEM measurements. A crosssectional HRTEM image obtained for the Er33 sample is presented in Fig. 4a. The micrograph shows that the matrix of the a-Si : $\mathrm{H}$ films contains a high density of small clusters, which were identified on the basis of electron diffraction analysis as silicon nanocrystals. The lattice fringes corresponding to the (111) planes of silicon are visible in the figure. Statistical analysis of the nanocrystallite size distribution gives a nanocrystal mean radius of about $1.5 \mathrm{~nm}$ for this sample. Note a PL response in the visible range at around $700-720 \mathrm{~nm}$ was also detected in these samples. We believe that, in our case, the situation is similar to that observed by Fujii et al. [15], who studied the correlation between the Errelated PL intensity and the nc-Si grain sizes in nc-Sicontaining $\mathrm{SiO}_{2}$ films doped with Er. These authors obtained a strong, by about two orders of magnitude, increase in the Er PL intensity with lowering the nc-Si grain size from 3.8 to $2.7 \mathrm{~nm}$. Actually, starting with these sizes, one can speak about a remarkable band-gap widening and the role of quantum size effects in Si [16]. Therefore, one can conclude that the seemingly strange increase in PL intensity in our "amorphous samples" can also be understood as a result of the sensitizing effect of Si nanocrystals on Er ions related to the enhancement of the excitation probability for the last ones due to the recombination of electron-hole pairs confined in nanocrystals. 


\begin{abstract}
4. Summary
In this contribution, we have demonstrated the possibility of producing, by the reactive magnetron sputtering method, effectively emitting nc-Si:H thin films doped with Er and have discussed their luminescent and structural properties. The nc-Si:Er films with welldefined crystallinity and nanocrystal sizes (varied from 1-3 to $8 \mathrm{~nm}$ ) were deposited and studied in both the near-IR and visible luminescence ranges. A strong influence of Si nanocrystals on the luminescent properties of the films at $1.54 \mu \mathrm{m}$ has been observed, where the most intense Er photoluminescence obtained relates to the low crystalline films with nanocrystal grain sizes less than $3 \mathrm{~nm}$. These films demonstrated intense $1.54 \mu \mathrm{m}$ luminescence at room temperature with a temperature- quenching coefficient (in the range $77-300 \mathrm{~K}$ ) of only about 5 . The results have been explained in terms of the sensitization effect of $\mathrm{Si}$ nanocrystals on Er rare-earth ions.
\end{abstract}

\title{
ACKNOWLEDGMENTS
}

This work was partially supported by the FCT foundation (Portugal) and the Russian Foundation for Basic Research (project no. 01-02-16439).

\section{References}

1. Silicon-Based Optoelectronics, Ed. by S. Coffa and L. Tsybeskov, MRS Bull., No. 23, 16 (1998).

2. J. Stimmer, A. Reittinger, J. F. Nützel, et al., Appl. Phys. Lett. 68, 3290 (1996).

3. B. Andreev, V. Chalkov, O. Gusev, et al., Nanotechnology 13, 97 (2002).

4. P. G. Kik and A. Polman, Mater. Sci. Eng. B 81 (1-3), 3 (2001).

5. F. Priolo, G. Franzo, F. Iacona, et al., Mater. Sci. Eng. B 81 (1-3), 9 (2001).

6. M. F. Cerqueira, J. A. Ferreira, and G. J. Adriaenssens, Thin Solid Films 370, 128 (2000).

7. M. F. Cerqueira, M. Andritschky, L. Rebouta, et al., Vacuum 46, 1385 (1995).

8. M. Losurdo, M. F. Cerqueira, E. Alves, et al., Physica E (Amsterdam) 16, 414 (2003).

9. R. Swanepoel, J. Phys. E 16, 1214 (1998).

10. I. H. Campbell and P. M. Fauchet, Solid State Commun. 58, 739 (1986).

11. M. Stepikhova, W. Jantsch, G. Kocher, et al., Appl. Phys. Lett. 71, 2975 (1997).

12. G. N. van Hoven, J. H. Shin, A. Polman, et al., J. Appl. Phys. 78, 2642 (1995).

13. G. Franzo, V. Vinciguerra, and F. Priolo, Appl. Phys. A 69, 3 (1999).

14. M. Losurdo, M. M. Giangregorio, P. Capezzuto, et al., Appl. Phys. Lett. (2003) (in press).

15. M. Fujii, M. Yoshida, S. Hayashi, and K. Yamamoto, J. Appl. Phys. 84, 4525 (1998).

16. T. Takagahara and K. Takeda, Phys. Rev. B 46 (23), 15578 (1992). 